\title{
Conceptualización del diseño de un sistema de georreferenciación participativo SIGP - como estrategia de comunicación política y responsabilidad social universitaria para la promoción comunitaria de la E-participación política.
}

\author{
Alonso Cortina Acevedo' \\ Bleidis Vanessa Quintana Pérez²
}

\section{Resumen}

En Colombia se entiende la participación ciudadana como un elemento democrático fundamental para la gobernanza y Desarrollo Humano Sostenible (DHS) de las comunidades, razón por la cual, la participación ciudadana se institucionaliza legalmente con la creación de los organismos de acción comunal, que por ley tienen la misión de ser articuladores del desarrollo comunitario, a través de la promoción de la

\footnotetext{
${ }^{1}$ Abogado especialista en derecho administrativo, especialista en gestión pública y doctor en ciencias políticas, investigador del grupo de investigación de la Universidad del Sinú Sociedad y Derecho,

${ }^{2}$ Abogada Especialista en Derecho Laboral y Seguridad Social. Magister en Cooperación Internacional para el Desarrollo. Docente investigadora adscrita al grupo de investigación Sociedad y Derecho de la Universidad del Sinú "Elías Bechara Zainúm" Seccional Cartagena.
} 
participación ciudadana, facilitando la comunicación política entre las autoridades del Estado en calidad de gobernantes y los ciudadanos como gobernados.

Dada la importancia constitucional que tienen los Organismos de Acción Comunal (OAC) frente al Desarrollo Humano Sostenible de la comunidad, el Estado implementa una política pública para el fortalecimiento de los OAC; sin embargo, y pese a los recursos públicos, que en este sentido se invierten, los organismos no logran incidir políticamente en la gestión pública. Según el diagnóstico de la politica pública efectuado a través de la presente investigación, esto se debe, entre otros factores, a la invisibilidad de los OAC, siendo una de las causas, la precaria participación ciudadana que los respalda, y el pobre desempeño que tienen como agentes comunicadores en los procesos de comunicación politica que los vinculan, como mediadores entre los ciudadanos y las autoridades del Estado.

Esta ponencia, presenta una estrategia de innovación social universitaria responsable (UNILAB) de la Escuela de derecho de la Universidad del Sinú "Elías Bechara Zainúm" Seccional Cartagena, que teniendo como criterios de diseño la informática comunitaria, la cartografía social, la responsabilidad social universitaria y la georreferenciación participativa, articula la universidad, los OAC, los ciudadanos y las autoridades públicas; Tal enlace se realiza a través de un circuito de comunicación politica, participativo y democrático, que dinamiza la e-participación ciudadana, fortaleciendo los procesos de visibilización sociopolítica de los organismos de acción comunal.

\section{Palabras claves}

Cartografía social; Comunicación política; E- Participación ciudadana, Georreferenciación participativa; Informática comunitaria; Responsabilidad Social Universitaria. 


\section{Abstract}

In Colombia, citizen participation is understood as a fundamental democratic element for the governance and Sustainable Human Development (DHS) of the communities, which is why citizen participation is legally institutionalized with the creation of community action agencies, which by law They have the mission of being articulators of community development, through the promotion of citizen participation, facilitating political communication between state authorities as governors and citizens as governed.

Given the constitutional importance of the Community Action Organizations $(\mathrm{OAC})$ in relation to the Sustainable Human Development of the community, the State implements a public policy for the strengthening of the OACs; However, and despite the public resources, which in this sense are invested, the agencies fail to influence politically in public management. According to the diagnosis of the public policy carried out through the present investigation, this is due, among other factors, to the invisibility of the CAOs, being one of the causes, the precarious citizen participation that supports them, and the poor performance they have as communicating agents in the processes of political communication that link them, as mediators between citizens and state authorities.

\section{Keywords}

Social mapping; Political communication; E- Citizen Participation, Participatory Georeferencing; Community informatics; University Social Responsibility. 


\section{Introducción}

La ponencia explica la primera fase de desarrollo de un software SIGP, sistema de información georreferenciado participativo, hecho a la medida. Esta etapa consiste en la conceptualización de la complejidad del problema a resolver y su contexto, de este ejercicio, resultan un conjunto de requerimientos que señalan las distintas funcionalidades que debe tener la herramienta tecnológica.

En el caso particular del SIGP UNILAB, la conceptualización del software consiste entonces en caracterizar y explicar el problema que representa para el desarrollo comunitario, la invisibilidad de los organismos de acción comunal; y justificar teóricamente, la selección de un sistema de información geográfica participativo como herramienta tecnológica que soporta el diseño de un circuito de comunicación politica, que promociona eficientemente, la e-participación ciudadana, visibilizando los organismos de acción comunal.

El trabajo se divide en dos partes, en la primera, se explica la invisibilidad politica de los OAC, como un problema que obstaculiza el Desarrollo Humano Sostenible de las comunidades. En este sentido, se caracteriza la relación que existe entre los OAC, el territorio, la comunicación politica, la participación ciudadana y el desarrollo de las comunidades; mostrando como la ineficiencia e ineficacia en los procesos de comunicación politica a nivel local barrial, afectan negativamente el empoderamiento territorial y con esto, la participación ciudadana, invisibilizando los OAC, restándoles capacidad para responder adecuadamente al rol de agentes articuladores de los proceso sociopolíticos de gobernanza y desarrollo comunitario. 
En la segunda parte, se presenta la conceptualización del proyecto UNILAB, como respuesta de solución a los problemas de empoderamiento territorial, comunicación politica y participación ciudadana, que invisibilizan a los OAC; en éste empeño, se explica el desarrollo teórico de las categorías conceptuales; responsabilidad social universitaria, informática comunitaria, cartografía social, e-participación ciudadana e información geográfica voluntaria; interrelacionándolas para explicar el diseño de un sistema de información georreferenciada participativo (en adelante SIGP), estructurado como un circuito de comunicación politica, que articula la academia, los organismos de acción comunal, los ciudadanos y las autoridades del Estado, con el fin de promocionar la e-participación ciudadana como estrategia de responsabilidad social universitaria que favorece la visibilización de los OAC.

EI SIGP pertenece a una categoría de herramientas tecnológicas, conocidas como las TEP, tecnologías para el empoderamiento y la participación, son aplicaciones diseñadas para la promoción de la participación ciudadana, en asuntos de interés político, social o educativo. Éste tipo de software, genera formas virtuales de interrelación de la población con su territorio y el poder político, la innovación social y educativa que se plantea, consiste en tomar el potencial que tiene el SIGP para producir sentido de pertenencia y participación, y ponerlo al servicio de los organismos de acción comunal, articulándolos con la universidad, para motivas dinámicas de reterritorrialización que faciliten procesos confiables de comunicación politica, que despierten en la comunidad la preocupación por los problemas de interés general y la participación ciudadana.

La articulación entre universidad y OAC, permite que éstos, con el apoyo de la proyección social, docencia e investigación de la responsabilidad social universitaria, desempeñen una representación politica efectiva en asuntos de control social, planeación participativa, diseño de presupuestos participativos, y otros procesos propios de la gobernanza comunitaria; luego valiéndose de UNILAB como circuito de comunicación politica, poder comunicar eficientemente toda esta actividad a las 
comunidades y procesar sistemáticamente sus inquietudes, exigencias y aportes, para retroalimentar a las entidades públicas, liderando procesos de legitimación o deslegitimación políticas de las acciones de gobierno. En consecuencia, se visibilizan los OAC frente a la comunidad y las autoridades de gobierno, lo que les permitirá desarrollar el papel cívico protagónico al que son llamados en el desarrollo comunitario.

\section{Metodología}

La invisibilidad de los OAC es una situación no provocada de manera intencional por los investigadores, de la que, además, se deprenden variables independientes y sobre las que no tiene, ni pretende tener control experimental el grupo de investigación. Consecuente con lo anterior, la investigación se diseña desde el paradigma cualitativo, interpretativo, con enfoque de investigación acción participativa, utilizando como técnica de análisis de datos fundamentada (Strauss y Corbin, 2004) estructurada en tres etapas o fases no lineales interactuantes entre sí, a saber, 1) Recolección de datos, 2) análisis de datos, y 3) formulación de teoría.

Para garantizar la validez del estudio, se apela en el diseño de la investigación al seguimiento de los criterios de validez de (Maxwell 1996), validez descriptiva, validez interpretativa y validez teórica, y la triangulación de métodos, de investigadores y de fuente de datos.

Siguiendo la metodología planteada, el diseño de UNILAB SIGP como circuito de comunicación politica, se concibe mediante el análisis de datos cualitativos de los cuales se evidenciaron categorías y conceptos como: comunicación politica, participación ciudadana, responsabilidad social universitaria, desarrollo comunitario, territorio, nuevas tecnologías, e-participación ciudadana, cartografía social, información georreferenciada participativa, tecnologías para el empoderamiento y la participación TEP, y organización comunal; reordenadas conformando un sistema categorial que explica la invisibilidad de los organismos de acción comunal, como un problema que afecta el desarrollo humano sostenible de las comunidades y que por lo tanto, es 
responsabilidad social de la universidad asumirlo como objeto de investigación aplicada.

Así mismo, con el sistema categorial expuesto se explica el diseño de un sistema de información geográfica participativo como herramienta TEP, idónea para estructurar un circuito local y comunitario de comunicación politica participativo, en el que a partir de la promoción de la e-participación ciudadana y la intervención de la universidad puede generar confianza en la intermediación politica que por ley deben hacer los organismos de acción comunal, entre la comunidad y las autoridades de gobierno.

\section{Desarrollo}

Para explicar la necesidad de dinamizar la participación ciudadana y la comunicación politica, como estrategia de visibilización y reconocimiento de los OAC, es menester iniciar por describir la relación que existe entre; la invisibilidad de los organismos de acción comunal, la participación ciudadana y la comunicación política.

\section{El problema de la invisibilidad de los organismos de acción comunal}

En Colombia la ley 743 de 2002 el desarrollo comunitario, describiéndolo como un conjunto de procesos económicos, políticos, culturales y sociales, que integran los diferentes actores del territorio, en pro de lograr la mejora continua de la calidad de vida de las comunidades, la misma norma, reconoce que el desarrollo comunitario, requiere la acción socio-politica de las organizaciones comunales, como medio adecuado para fortalecer la participación politica de la comunidad en los procesos de desarrollo humano y sostenible de su territorio.

La ley 743 de 2002 define las juntas de acción comunal, como organismos de acción comunal, de naturaleza solidaria, dispuestos para la gestión cívica, social y comunitaria, integrados voluntariamente por los residentes de una localidad, que se solidarizan en pro de lograr un desarrollo integral, sostenible y sustentable, ejercitando acciones de democracia participativa; para mejor ilustración, se reseñan algunas de las 
principales funciones de los $O A C$, que permiten dimensionar el alto impacto que la invisibilidad de estos puede tener, en el desarrollo comunitario.

Son funciones de los organismos de acción comunal, artículo 19, ley 723 de 2002:

- Promover y fortalecer el sentido de pertenencia y la democracia participativa

- promocionar el ejercicio de la democracia

- Establecer canales de comunicación necesarios para Lograr que la comunidad esté permanentemente informada sobre el desarrollo de los hechos, políticas, programas y servicios del Estado y de las entidades que incidan en su bienestar y desarrollo Generar procesos comunitarios autónomos de identificación, formulación, ejecución, administración y evaluación de planes, programas y proyectos de desarrollo comunitario;

- Recuperar y fortalecer la identidad comunal;

- Construir y preservar la armonía en las relaciones interpersonales y colectivas en el territorio de su jurisdicción,

- Velar por una mejora constante de la calidad de vida en su jurisdicción;

Como se puede apreciar, para la comunidad es un problema grave la invisibilización de los OAC, porque estos organismos como expresión de participación ciudadana institucionalizada, tienen la función de velar por la promoción de procesos vitales para el desarrollo humano y sostenible como: la comunicación politica, la cohesión social, la identidad colectiva, el sentido de pertenencia y la participación ciudadana, todos éstas, categorías relacionadas con la búsqueda, colectiva y solidaria, de soluciones a los problemas de interés común en el territorio, de manera que cuando los OAC se invisibilizan, se deteriora la comunicación entre los órganos del Estado y las comunidades, dificultándose para el ciudadano la posibilidad de conocer la complejidad de los problemas que afectan colectivamente el territorio donde residen, sobreviniendo con esto, la perdida de interés por la participación ciudadana. 
El territorio se constituye por ser un fuerte elemento condicionador de la forma en que política y socialmente se concibe a sí mismo el sujeto en su relación con el Estado y la sociedad, esto en la medida en que tal como dice Ana Maria Portal:

Las identificaciones sociales se construyen a partir de experiencias concretas, históricamente determinadas, que pueden variar en el tiempo y que se traducen en preguntas tales como: ¿cómo me ven los otros?, ¿cómo me nombran?, ¿cómo me definen? Y de esas identificaciones, ¿con cuáles me quedo?, ¿cuáles me sirven para definirme y cuáles no?(Portal, 2003, pág .23)

Según Portales, un territorio deteriorado no podrá proporcionarle a quien lo habita experiencias de vida que permitan construir una relación adecuada entre el sujeto y su entorno, en consecuencia, si el territorio se deteriora se deteriora igualmente la identidad social urbana, afectándose el sentido de pertenencia con el territorio, produciéndose la pérdida de imaginarios comunes que permitan trabajar en pro del interés común, deteriorándose el tejido social al punto de llegar casi a desaparecer la participación ciudadana, afectándose con esto los procesos democráticos.

La ausencia de participación ciudadana, invisibiliza para la administración los problemas locales que comúnmente afectan al barrio como territorio, sobreviniendo su deterioro material y socio-político que lleva finalmente a la pérdida de control del territorio, produciéndose la desvinculación del sujeto con su entorno físico y con los demás, fenómeno conocido como desterritorialización.

Como se explicaba anteriormente, la falta de control del territorio conlleva a la perdida de sentido de pertenencia, al desarraigo y debilitamiento de la identidad colectiva (Durán, 2009) la cual, a nivel comunitario, podríamos definir como identidad barrial; sin una identidad común, que vincule los intereses materiales y emocionales de los residentes del barrio. Se diluye la posibilidad de trabajar mancomunadamente por los problemas que afectan a la comunidad, y entonces, el sujeto antes que percibir su territorio como una fuente de respaldo político y social, que proporciona soluciones a 
los problemas particulares y colectivos, lo entiende y rechaza como una amenaza de la que debe defenderse con sus propios recursos, desconfiando de toda propuesta de cambio que proceda de su mismo entorno y las autoridades responsables de la preservación o deterioro del mismo.

\section{De la relación entre comunicación política, las nuevas tecnologías y la invisibilidad de los organismos de acción comunal}

La comunicación politica con el desarrollo vertiginoso de la web 2.0, dejo atrás el modelo masivo unidireccional en el que los ciudadanos, eran consumidores de la información que el Estado proporcionaba de su gestión de gobierno, sistema soportado mayormente por canales de comunicación masiva como la televisión y la prensa, agotado por despotismo, la comunicación politica se democratizó, la web 2.0, convirtió al ciudadano en "prosumidor" (Carmona, 2008, pág. 15), de información o mensajes con impacto sociopolítico.

En la era digital el ciudadano no sólo consume información politica, también la produce, y con esto impacta la gestión pública, con tal grado de incidencia, que ahora la acción de gobernar debe necesariamente contar con canales de comunicación politica de doble vía, ya no puede aislarse elitistamente, como sucedía en el modelo de comunicación politica broadcasting, en el que el agente político, sólo contaba con la movilización politica de los ciudadanos en momentos electorales, pero luego, se aislaba en su quehacer como gobierno, sin que el ciudadana tuviese mayores oportunidades de ser escuchada y mucho menos de participar en la planeación, ejecución y control de la gestión de gobierno.

Con el surgimiento de la politica 2.0, la e-democracia y la e-participación ciudadana, emerge una realidad virtual que no permite ejercicios de gobernanza que no tengan en cuenta el mejoramiento de los canales y medios de comunicación politica 2.0., de ahí, que pretender reducir la comunicación politica a la información o publicidad 
politica pagada en medios, privatizando las representaciones y decisiones políticas; resulta ser desacertado por el desconocimiento que se hace del derecho de la ciudadanía de manifestarse, haciendo además, altamente manipulable las concepciones políticas de los ciudadanos y su consecuente participación pública (Cisneros, 2006).

Así las cosas, los OAC como instituciones de participación ciudadana organizada, para visibilizarse, requieren canales de comunicación politica vinculados con la web 2.0. y la e-participación ciudadana, toda vez que, amen de lo dicho anteriormente, recordemos que la ley 743 en el artículo 19, les encomienda la tarea de establecer canales de comunicación con los cuales la comunidad esté permanentemente informada sobre el desarrollo de los hechos, políticas, programas y servicios del Estado y de las entidades que incidan en su bienestar impactando programas y proyectos de desarrollo comunitario.

Conforme a lo anterior, los OAC deben ser mediadores en dinámicas de comunicación politica, intentando encontrar un mejor entendimiento entre los diferentes estamentos que conforman la sociedad y entre ésta y el grupo gobernante, por lo tanto, si el desarrollo de las nuevas tecnologías, como las TEP, implican la democratización de la comunicación politica, mediante la apertura a la participación ciudadana, es de obligada ocurrencia, la incursión de los OAC en procesos de e-participación ciudadana, haciendo uso de tecnologías que promocionan la participación y el empoderamiento político de la población para lograr así visibilizarse ante la comunidad y las autoridades de gobierno.

\section{Comunicación política participativa y responsabilidad social universitaria.}

Como se dijo anteriormente, la comunicación política actualmente, se complejiza por el avance vertiginoso de las herramientas tecnológicas en la web 2.0, disponibles y de fácil acceso en internet que permiten al ciudadano promedio, consumir y producir 
información politica, en una nueva categoría de espacio público virtual, en el que la transmisión de mensajes entre partidos políticos y ciudadanos, o bien entre mandatarios y gobernados, se realiza con frenesí en tiempo presente, caracterizando lo que hoy se conoce como e-participación ciudadana asociada directamente con la comunicación politica participativa (Ugarte, 2019).

Pese a lo anterior, si bien es cierto, la web 2.0, democratizó los procesos de comunicación politica, aperturándolos a la participación ciudadana, no es menos cierto que la información politica que se produce y consume en la web 2.0, tiene un alto déficit de confiabilidad, por cuanto la generalidad es que la fuente que la produce, carece de respaldo profesional, académico y científico, lo que además, la hace altamente vulnerable a la manipulación por parte de intereses particulares que la utilizan para generar, malintencionadamente, opinión publica favorable o desfavorable a determinado proceso, proyecto o consigna politica.

Siendo, así las cosas, para que los OAC se vinculen con la web 2.0, como canal de comunicación politica con potencial para motivador de la e-participación ciudadana, se requiere que la información socio-politica que éstos median, tenga respaldo profesional, académico y científico, dotando de mayor confiabilidad y eficacia los procesos de comunicación politica participativa, que dependen del ejercicio legal del rol cívico - político de los organismos de acción comunal.

Con todo esto, la pregunta entonces sería, ¿Qué actor o institución con capacidad para impactar el desarrollo local humano y sostenible de la comunidad, tiene el suficiente respaldo político y la credibilidad social para respaldar y promocionar las dinámicas de comunicación politica de los organismos de acción comunal?

Sin duda alguna, la respuesta la encontramos en la responsabilidad social universitaria, la Universidad como actor social, está llamada a ser protagonista en el desarrollo territorial endógeno, motivando en los actores con mayor capacidad de impacto en el el desarrollo comunitario, la defensa coordinada del intereses general, 
identificando y potencializando o construyendo, las ventajas competitivas y comparativas que permitan direccionar procesos de productividad, competitividad y asociatividad que dinamicen del desarrollo humano y sostenible de la sociedad.

El proyecto UNILAB SIGP como circuito de comunicación politica participativa, es el resultado de una estrategia educativa innovadora, mediante la cual la Universidad del Sinú "Elías Bechara Zainúm" Seccional Cartagena, asume la responsabilidad social que tiene frente al desarrollo integral de sus estudiantes como futuros profesionales socialmente responsables y el desarrollo local, humano y sostenible de la comunidad.

En este sentido, vale resaltar, que en la modernidad última, la responsabilidad social universitaria, ha tenido gran debate por el poco impacto que la universidad tiene en la solución de problemas y conflictos sociales, exigiendo por esto la sociedad, una institución de educación superior, más dedicada a la búsqueda de soluciones a problemas cotidianos, más comprometida con las diferentes realidades políticas, la defensa de la democracia y los derechos humanos, la erradicación de la pobreza, la preservación del medio ambiente entre otros.

El Instituto Internacional de la UNESCO para la Educación Superior en América Latina y el Caribe (IESALC) junto con diversos autores, concuerdan en concebir las universidades como instituciones de gran impacto político y social, que deben involucrarse responsablemente en la transformación de las estructuras de injusticia y desigualad de las sociedades, innovando socialmente desde los ámbitos que le son propios, docencia, investigación, y extensión (Yarzábal, 1999).

El profesional debe estar al servicio de la sociedad y conocer las circunstancias que lo rodean desde el plano económico, político, social y cultural, buscando constantemente la solución de problemas identificados y evidenciando los resultados del aprendizaje en su ejercicio profesional por medio de la innovación (Tornés, Rivera y Téllez, 2019). 
Como centro de producción de conocimiento e innovación social, y tecnológica, la universidad, tiene la capacidad instalada para abordar la complejidad de la comunicación politica participativa, y como ejercicio de investigación aplicada, en asocio con los organismos de acción comunal, diseñar estrategias, programas y proyectos que la doten de mayor confiabilidad y eficacia.

\section{Diseño conceptual de UNILAB SIGP como circuito de comunicación política participativa}

Siguiendo el método de investigación acción participativa, el diseño de UNILAB SIGP se concibe en asocio con la Federación de Acción Comunal del Distrito de Cartagena, organización comunal de tercer nivel, que representa los niveles 1 y 2 de toda la estructura de acción comunal institucionalizada localmente en el distrito de Cartagena. Así, se conformó una comunidad de aprendizaje mutuo, conformada por la universidad, representada por los docentes investigadores, estudiantes asignados al proyecto, por los lideres designados por la federación de acción comunal.

En este contexto de enseñanza aprendizaje colaborativo, los dignatarios de la federación de acción comunal, los docentes y estudiantes, durante el periodo de un año, fueron instruidos mediante seminarios en los que se trataron temas como la metodología de la investigación acción participativa, la inteligencia colectiva territorial, ciudad inteligente, comunicación politica, desarrollo comunitario, democracia y participación ciudadana,

Durante el proceso de capacitación, al mismo tiempo que los estudiantes y líderes fueron aprendiendo conocimiento académico científico; mediante entrevistas y encuesta realizadas a los dignatarios de la federación y de las juntas de acción comunal, se capitalizó el conocimiento empírico que los OAC poseen sobre los problemas de representación y comunicación politica que éstos tienen por la falta de respaldo institucional, capacitación y recursos tecnológicos. 
En consecuencia, desde la emergencia de un nuevo conocimiento colaborativo se concibió la innovación social y tecnológica UNILAB SIGP, como idea de innovación social y tecnológica apropiada socialmente desde el inicio del proyecto.

UniLab se constituye en un sistema de información georreferenciada participativo estructurado como un circuito de comunicación politica participativa, gestionado por docentes, estudiantes y dignatarios de organismos de acción comunal, previamente capacitados en el manejo de la información que produce y recibe el sistema a través de una plataforma y aplicación virtual.

Como circuito de comunicación política, Unilab, bajo la coordinación científica y académica de la universidad, articula, gestiona y procesa la información que proviene de las autoridades de gobierno, de los ciudadanos que hacen uso de la plataforma virtual y de la APP, haciendo uso de técnicas de cartografía social y georreferenciación geográfica participativa, la información es plasmada en mapas del territorio que representan su realidad vista a partir de quienes lo residen o resultan afectados por los problemas que esté presente, de ésta manera se pone en contacto la universidad y los organismos de acción comunal con los ciudadanos.

Unilab configura dinámicas de comunicación politica porque facilita el reporte incidentes y problemas urbanos de diferente índole, recopilando información importante para su gestión y solución, tales como datos geográficos y de geolocalización, tipología, nivel de riesgo, imagenes, videos y descripción detallada del incidente. Esto con el fin ser asignado automáticamente, a cada caso, a un equipo de trabajo multidisciplinar conformado por líderes comunales, estudiantes y ciudadano que reporta el incidente, si así lo desea, una vez recibida la información el equipo al que se le asigna la analiza y si encuentra que es real se notifica al ciudadano el inicio de las diligencias pertinentes y necesarias para resolver el problema o incidente.

El sistema crea un base de datos con toda la evidencia (documental, fotográfica, videos, conversaciones, etc) que se genere en el tramite del incidente, haciendo visible 
púbicamente la trazabilidad de la gestión informando la identidad del dignatario de acción comunal que la coordina y las autoridades involucradas en la gestión del incidente, las respuestas, requerimientos y soluciones que éstas puedan dar al problema planteado, cuando el incidente puede ser resulto mediante la acción comunitaria de los vecinos del sector, el dirigente comunal con el apoyo de la proyección social de la universidad, entrará a diseñar un proyecto comunitario para presentar a los vecinos la solución, toda ésta gestión tendrá una trazabilidad verificable mediante la aplicación web mediante notificaciones que se reciben en tiempo real.

De la gestión de incidente, el sistema genera reportes estadísticos con la información generada por la interacción ciudadana alrededor de los problemas o incidentes que ocurren dentro del seno urbano de la ciudad, dicha información estadista es de suma importancia para el cumplimiento eficiente del rol socio - politico de los OAC, en la medida de que les permite tener un conocimiento avanzado de los problemas del territorio de su jurisdicción, lo que facilita ejercicio de gobernanza participativa como el control social de la gestión del gobierno, la planeación participativa del presupuesto local, la gestión del riesgo, las condiciones de seguridad ciudadana, movilidad e integridad del espacio público, teniendo datos que provienen directamente del ciudadano afectado, diseño de programas y campañas de acción comunal para el mejoramiento del territorio barrial.

Todas éstas posibilidades tienen el potencial de generar capital social, en la medida en que las personas tienen oportunidad de mantener contacto permanente con la problemática del territorio, haciendo seguimiento al avance y resultado de las acciones que los ciudadanos proponen, esto hace que se active un proceso de recuperación de la participación ciudadana por la reterritorrialización que motiva el sistema.

Desde el punto de vista técnico, Unilab está desarrollada inicialmente para ser utilizada desde dispositivos móviles SmartPhone con Sistemas Operativo Android minima 2.0 en adelante. Su arquitectura está basada en la integración de múltiples, articulación y comunicación herramientas online, en su gran mayoría basadas en 
tecnología WebServices RESTful. entre otras: Servicio de Almacenamiento de datos, servicio de envío de Correos; servicio de Notificaciones Push en tiempo real; servicio de geolocalización y georreferenciación; servicio de integración con redes sociales.

Así mismo, realiza una integración armónica entre dichos servicios de tecnología optimizando los limitados recursos de datos, pantalla y almacenamiento que poseen la mayoría de dispositivos móviles, pero al mismo tiempo del mercado, aprovechando a su vez, el uso masivo a nivel global, conectividad, portabilidad, capacidades de cálculo, integración al contexto y la practicidad que caracterizan estos dispositivos, todo con el fin de ofrecer una solución muy útil para mejorar de forma participativa y colaborativa la comunicación politica entre ciudadanos, organismos de acción comunal y las autoridades de gobierno.

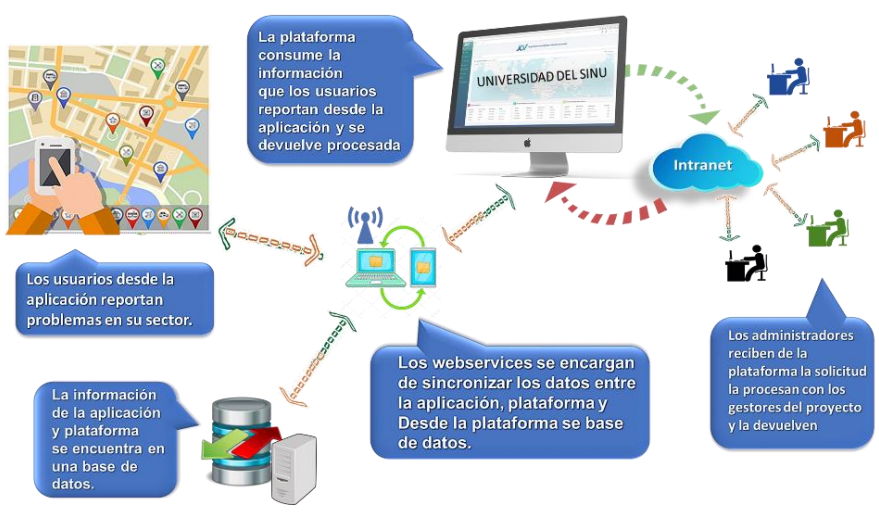

Gráfica 1. Diagrama de contexto. Elaboración de Autores. 
La base de datos que el sistema va alimentando con cada incidente que se reporta, está disponible para todos los actores del circuito, ciudadanos, autoridades públicas, docentes investigadores, estudiantes, todo actor o institución con capacidad de transformación del territorio que quiera vincularse de manera permanente al proyecto. En ese sentido, a través de la gestión de la federación de acción comunal, varias universidades con sede en el distrito de Cartagena, han pedido información del proyecto y el código de fuente del software para vincularse generando más investigación que proporcione más y mejores utilidades para el sistema, así mismo, para asumir el rol de coordinación de equipos de gestión de incidentes.

UNILAB SIGP cuenta con un plan de mejora continua, en el que se plantea periódicamente la capacitación en la gestión y operación del sistema a nuevos actores, como por ejemplo, instituciones civiles de primer orden, como asociaciones de vecinos, fundaciones, sindicatos y ONGs, entre otras, interesados en integrarse al circuito de comunicación politica. La idea es que entre más diversos sean los sectores sociales que se vinculen a al sistema mejorará la visibilidad de los OAC.

\section{Conclusiones}

El diseño del proyecto UNILAB muestra como conceptualmente las dinámicas de comunicación politica participativa mediadas por la web. 2.0, se complejizaron a tal punto, que se constituyeron en un problema para el desarrollo humano y sostenible, problema que no puede ser resuelto por la politica pública de participación ciudadana y fortalecimiento de los organismos de acción comunal, mientras la universidad no asuma su responsabilidad social frente el tema. 
Las nuevas tecnologías para el empoderamiento y la participación -TEP, deben ser más investigadas y desarrolladas, puesto que cada vez con mayor intensidad la comunicación politica del ciudadano con el gobierno, se da mediante la web 2.0, si las universidades no asumen el reto de vincularse en las dinámicas de desarrollo humano y sostenible, asumiendo decididamente un rol político, la web 2.0 antes que fortalecer la comunicación politica la deteriorará, abriendo la brecha aún más, entre quienes tienen acceso a información politica de calidad y quienes asumen posiciones políticas manipulados por la confusa comunicación politica que hoy transita en la red.

\section{Referencias Bibliográficas}

Becerra Brito, C. V. (2019). El uso de TIC, TAC y TEP: Una asignatura aún pendiente para el profesorado de inglés. Recuperado de: https://riull.ull.es/xmlui/bitstream/handle/915/16809/El\%20uso\%20de\%20TIC,\%2 0TAC\%20y\%20TEP\%20\%20Una\%20asignatura\%20aun\%20pendiente\%20para $\% 20$ el\%20profesorado\%20de\%20ingles.pdf?sequence $=1$

Carmona, J. O. I. (2008). El prosumidor. El actor comunicativo de la sociedad de la ubicuidad. Palabra clave, 11(1). Recuperado de: https://educacionyeducadores.unisabana.edu.co/index.php/palabraclave/article/vi $\underline{\text { ew/1413/0 }}$

Cisneros, J. (2006). El debate político como patrimonio público. Revista Latina de Comunicación Social, 9(61), $0 . \quad$ Recuperado de: https://www.redalyc.org/pdf/819/81996102.pdf

Durán, F. (2009). La desterritorialización de las comunidades locales rurales y su creciente consideración como unidades de desarrollo. Revista de desarrollo rural y cooperativismo agrario, 3. Recuperado de: http://www.academia.edu/download/57550773/Desterritorializacion Rural.pdf

Esteban, M. P. S. (2000). Criterios de validez en la investigación cualitativa: de la objetividad a la solidaridad. Revista de investigación educativa, 18(1), 223-242. 
Portal, M. A. (2003). La construcción de la identidad urbana: la experiencia de la pérdida como evidencia social. Alteridades, (26), 45-55. Recuperado de: https://alteridades.izt.uam.mx/index.php/Alte/article/view/323

Strauss, A., \& Corbin, J. (2016). Bases de la investigación cualitativa: técnicas y procedimientos para desarrollar la teoría fundamentada. Universidad de Antioquia.

Ugarte Iturrizaga, A. (2019). LA PARTICIPACIÓN COMO ESTRATEGIA DE COMUNICACIÓN POLÍTICA. Recuperado de: http://redmarka.net/ra/revista. php? wid=9\&articulo=576\&tipo=\&eid $=22 \&$ sid $=152 \&$ NombreSeccion=Articulos\&Accion $=$ Completo

Ugarte Iturrizaga, A. (2019). LA PARTICIPACIÓN COMO ESTRATEGIA DE COMUNICACIÓN POLÍTICA. Recuperado de: http://redmarka.net/ra/revista. php? wid=9\&articulo $=576 \&$ tipo $=\&$ eid $=22 \&$ sid $=152 \&$ NombreSeccion=Articulos \&Accion $=$ Completo

Yarzábal, L. (1999). Consenso para el cambio en la educación superior (Vol. 9). lesalc/UNESCO. 